\title{
Conventional versus Flap Tracheostomy: Operative Simplicity, Stomal Care, Complications
}

\author{
https://doi.org/10.47210/bjohns.2020.v28i2.328
}

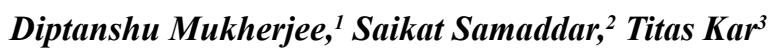

\section{Introduction}

\section{ABSTRACT}

Tracheostomy is a life saving procedure and its operative principle has withstood the test of time although the operative techniques have evolved. Inferiorly based flap on the anterior tracheal wall (Bjork flap) was demonstrated in 1952. The present study aims to compare conventional tracheostomy with flap tracheostomy.

\section{Materials and Methods}

ARandomized Control Trial was conducted in a tertiary care teaching medical institute comparing conventionally tracheostomised patients (Group A) with the group where flap based method was followed (Group B).

\section{$\underline{\text { Results }}$}

The study population was comprised of 110 patients with equitable distribution in the groups. Per-operative time to establish an airway was measured and statistically correlated. 9.26\% patients of Group A had difficult tube change, with none in Group B. Ease of stomal care by the patient and family members (Visual Analogue Scale), depicted it to be "very easy" in $76.36 \%$ of the patients in Group B and 16.36\% in Group A. Stomal healing in first week was proper in $61.82 \%$ of patients in Group A and $80 \%$ of the patients in Group B. Tracheo-cutaneous fistula (TCF) developed in $18.18 \%$ of the adult and geriatric patients of Group A undergoing stomal closure. None of the patients in Group B had TCF following stomal closure.

\section{Conclusion}

Establishing Bjork flap tracheostomy is equally quick making the process suited for emergency situations. Flap based tracheostomy has early stomal maturation so, care of the stoma by health assistants and family members is easy.

\section{$\underline{\text { Keywords }}$}

Tracheostomy; Surgical Flap, Anterior Tracheal Wall

$\mathrm{T}$ Tracheostomy has been a life saving procedure practiced since time immemorial. ${ }^{1}$ Chevalier Jackson in the twentieth century defined the principles of the operation. ${ }^{2}$ Since then the indications have evolved. So has evolved the operative techniques sticking to the same operative principles. Literature has

1 - Department of ENT, Medical College and Hospital, Kolkata.

2 - Department of ENT, Deben Mahato Government

Medical College and Hospital, Purulia.

3 - Department of ENT, IPGME\&R, SSKM Hospital,

Kolkata.

Corresponding author:

Dr Saikat Samaddar

email: saikat12@gmail.com documented procedures ranging from making a surgical opening in the trachea via an incision on the tracheal wall (tracheotomy), excision of portion of the anterior wall cartilage (tracheostomy) to facilitate the stoma, flaps of the tracheal wall for ostomy to percutaneous dilatation methods under endoscopic guidance. Amongst the various flaps of the anterior tracheal wall that has been advocated, inferiorly based tracheal flap demonstrated by Bjork in 1952 has stood the test of time. ${ }^{3}$ Specific complications have been attributed to Bjork tracheostomy though the same has been a matter of debate. ${ }^{4}$

The present study aims to determine the operative simplicity in establishing a flap tracheostomy as compared to conventional method. Assess the post operative ease of stomal care delivered by trained 
nurses and family members. Evaluate compliance of Bjork Flap Tracheostomy with respect to conventional Tracheostomy.

\section{Materials and Methods}

A Randomized Control Trial was conducted in a tertiary care teaching medical institute of Kolkata, West Bengal, from August 2014 to July 2016. Patients requiring tracheostomy for establishing the airway were considered as the universe. Among them, those patients undergoing tracheostomy in a fixed surgical unit of the institute under the same senior surgeon were only included in the study. The study population were divided into two groups (i.e. Group A and Group B) by Block Randomisation with a Block of 4. Single blinding was done where the patient consented for both the procedures but didn't know, to which group they will be allotted to.

The study population was comprised of 110 patients $(n=110), 55$ were allotted in either group. The age range of the study population was 4 to 75 years.

Patients undergoing tracheostomy with neoplasm on the tracheal wall, intra-luminal growth, deviated trachea, prior tracheostomised state were excluded from the study.

In Group A, the adult patients underwent conventional tracheostomy. Following a midline vertical skin incision ranging from the level of the lower border of the cricoids cartilage to the suprasternal notch, soft tissue was dissected and strap muscles retracted to expose the trachea. Thyroid isthmus was retracted if required. Anterior wall of the tracheal cartilage was excised ranging from the level of the $3^{\text {rd }}$ tracheal ring to the $4^{\text {th }}$ tracheal ring. In prior intubated patients the endotracheal tube was removed before high volume low pressure cuffed Portex ${ }^{\mathrm{TM}}$ tracheostomy tube was placed. In paediatric patients undergoing tracheotomy, no tracheal cartilage was excised. A vertical midline incision was placed on the anterior tracheal wall after placing stay suture on the either sides. Age appropriate proper size tracheostomy tube was placed across the incision. Local dressing was done as per protocol.

In Group B, Bjork flap tracheostomy was done.
Vertical midline incision was placed similar to conventional method. Further dissection was done to expose the trachea. An inferiorly based flap (inverted ' $U$ ' shapped) was fashioned on the anterior tracheal wall. The superior limit of the flap was an inter cartilaginous horizontal incision between the second and the third tracheal rings. On either side, the horizontal incision was turned down cutting through the third and the fourth rings, hinging across a base of around $12-15 \mathrm{~mm}$. In paediatric patients the similar inferior based anterior tracheal flap was made with base width of around $5 \mathrm{~mm}$. The upper lip of the flap was secured to the lower border of the skin incision with 2-0 synthetic, monofilament, non-absorbable polypropylene suture. Age appropriate proper size tracheostomy tube as used in group A was placed. Local dressing was done.

Per operatively parameters such as, ease of the procedure, time required to establish airway, difficulties encountered and complications resulted were noted.

The patients were followed up for one year. Institution based protocol for tracheostomy care was observed. First tube change was performed at 48hours. Next change at 7 days or as and when required. During the follow-up period, documentation was made as to the ease of stomal care when done by the trained nursing personnel during the first tube change and subsequently by the patients or family members. A Visual Analogue Scale was utilised to evaluate the same. Post operative complications during the follow-up period were noted. All patients including those undergoing stomal closure went through flexible endoscopic evaluation of trachea during the closure procedure and subsequently at 4 months interval to exclude tracheal stenosis. For conventional tracheostomy closure (Group A), the stoma was refashioned. Adjacent neck muscle layer was dissected, isolated and closed in midline followed by midline skin closure. In Group B, the inferiorly based flap was released to ensure closure. Tracheal cartilage flap was repositioned but not sutured to the tracheal wall. The muscle and the skin were closed in midline separately.

\section{Results}

The demographic analysis of the study population 
Table I: Distribution of study population in various age groups

\begin{tabular}{|c|c|c|}
\hline & GROUP A & GROUP B \\
\hline PEDIATRIC (4-12yrs) & 1 & 3 \\
\hline ADULT (13-60yrs) & 27 & 25 \\
\hline GERIATRIC (61-75yrs) & 27 & 27 \\
\hline
\end{tabular}

revealed, $72.72 \%$ of the study population was male and the rest female. Both the genders were equally distributed in either group. Paediatric (4-12years), adult (13-60years) and geriatric population (60-75years) in Group A was $1.81 \%, 49.09 \%$ and $49.09 \%$ respectively. The distribution of ages in Group B was 5.45\%, 45.45\% and $49.09 \%$ respectively. (Table I)

In Group A, planned tracheostomy was done in $23.63 \%$ patients and the rest underwent emergency tracheostomy. In Group B, 30.91\% underwent planned surgery and the rest emergency intervention. In Group $\mathrm{A}$ and $\mathrm{B}, 7$ and 12 patients respectively had received radiation therapy prior to tracheostomy. (Fig. 1)

Per-operative time required to establish an airway was measured in both the groups. It was classified as 'under 4 minutes', '4-8 minutes' and 'more than 8 minutes'. In Group A, airway was established in 4 patients under 4minutes, 44 patients in 4-8 minutes and 7 patients required more than 8 minutes. In Group B, 5 patients had established airway in 4 minutes, 49 patients in 4-8 minutes and 1 required more than 8 minutes. Statistical correlation was done amongst Group A and B. Chi square value was found to be 4.879. $\mathrm{p}$ value of 0.087 $(>0.05$, i.e. not significant).

During the post operative period the first tracheostomy tube change is done by trained nursing personnel along with an ENT surgeon 2 days after the surgery. Any prior tube change is done solely by team of surgeons. The first protocol based tube change at 48 hours post operative was termed difficult if the nursing personnel required active intervention by the ENT surgeon during such procedure. In Group A, the single paediatric patient who underwent tracheotomy had a difficult first tube change. 5 adult patients $(9.26 \%)$ of Group A had difficult tube change. None of the patients in Group B required active intervention of an ENT surgeon during the first tube

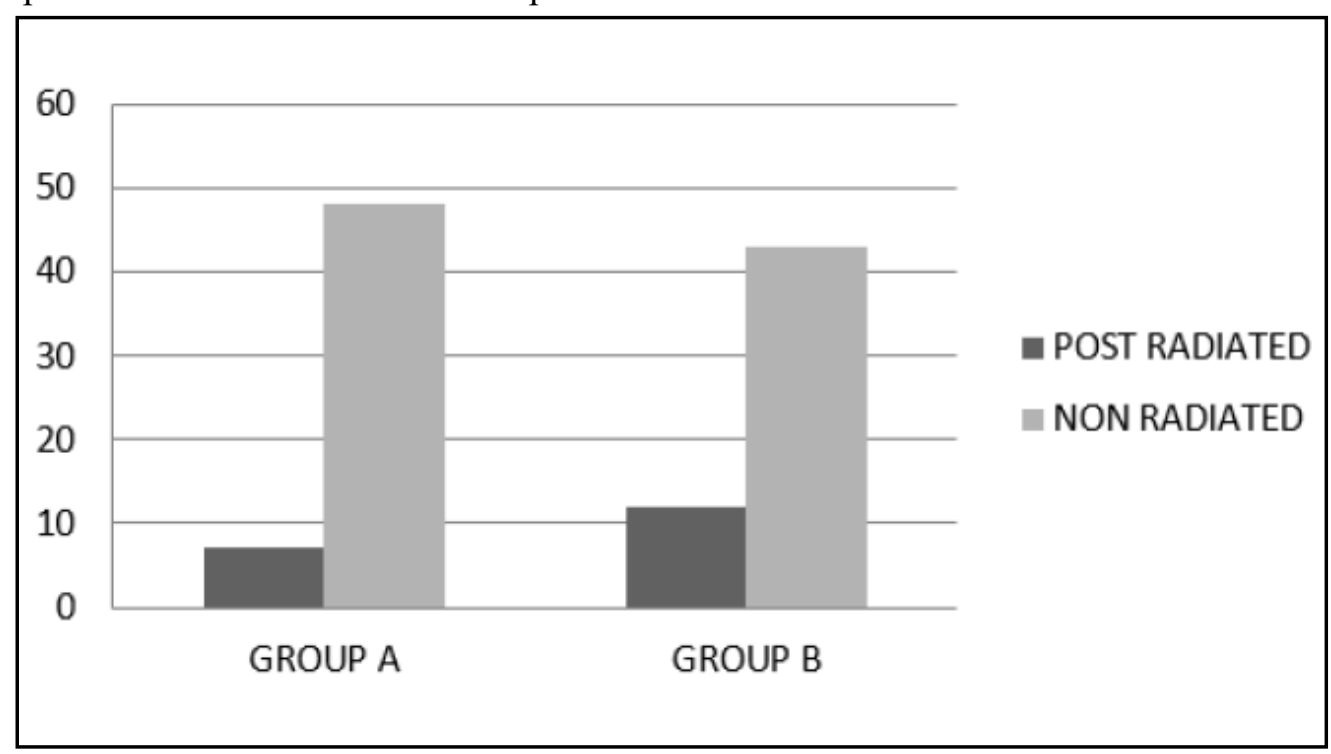

Fig. 1. Distribution of patients according to pre operative radiation exposure 
Table II: Distribution of patients in either group in matter of stomal care ease by patients and family members, assessed by Visual Analogue Scale score

\begin{tabular}{|c|c|c|c|}
\hline & GROUP A & GROUP B & TOTAL \\
\hline VAS 0 & $9(16.363 \%)$ & $42(76.363 \%)$ & 51 \\
\hline VAS 1 to 10 & 46 & 13 & 59 \\
\hline TOTAL & 55 & 55 & 110 \\
\hline
\end{tabular}

Chi -39.8106

$P$ - $<0.00001$ Significant difference

change.

Further the ease of tracheostomy care by the patients and family members was assessed by a Visual Analogue Scale, ranging from "0"- Very easy to "10"- Very difficult. In Group A $16.36 \%$ of the patients reported stomal care to be "very easy". $76.36 \%$ of the patients in Group B reported it to be "very easy". Statistical correlation showed Chi square value of 39.81. $\mathrm{p}$ value of $<0.00001$. $(<0.05=$ significant difference). (Table II)

Stomal healing within first week was proper in $61.82 \%$ of patients in Group A and $80 \%$ of the patients in Group B.

Closure of tracheal stoma was tried in all the paediatric patients belonging to both the groups. In adult and geriatric population it was tried in $40.74 \%$ patients in Group A and 55.77\% patients in Group B. Average time of stomal closure was 2 weeks following tracheostomy. In Group A, the only child undergoing tracheotomy had resulted Tracheo-Cutaneous Fistula (TCF) following stomal closure. $18.18 \%$ of the adult and geriatric patients of Group A undergoing stomal closure had TCF. None of the patients in Group B had TCF following stomal closure.

As mentioned, tracheal stenosis was evaluated in all the patients, specifically those undergoing stomal closure. Stenosis was evaluated at the level of the stoma and at the level of the tip of the tracheostomy tube. In either group no cases of tracheal stenosis was found during the follow up period.

Other complications encountered both per operatively and post operatively was evaluated. Primary haemorrhage was found in 5 patients of Group A and 4 in Group B. Secondary haemorrhage, 2 in Group A and nil in Group B. 4 patients had surgical emphysema in Group A and only 1 patient in Group B. Dysphagia was complained by 2 patients in Group A and 1 patient in Group B. Peristomal granulation, stomal stenosis, trachea-esophageal fistula and trachea-cutaneous fistula was found in 4, 6, 1 and 5 patients respectively in Group A. None of these complications were present in any of the patients of Group B. Tracheostomy related death didn't occur in either group. (Fig. 2)

\section{Discussion}

Tracheostomy is a common procedure in an ENT operating room and intensive care units. The planned tracheostomies were performed prior to major head neck and maxilla facial surgeries. Few patients of laryngeal carcinoma put to radiotherapy were tracheostomised considering impending airway obstruction following radiation induced oedema. Patients requiring prolong mechanical ventilation were also tracheostomised. All the paediatric patients in the study population underwent tracheostomy as an indication for prolong mechanical ventilation. Emergency tracheostomies were performed in cases of laryngeal neoplasm with stridor, maxillofacial and airway trauma, endolaryngeal foreign body.

Midline vertical skin incision has been used in both the groups undergoing tracheostomy. This elaborate incision offers better access, adequate visualisation of deeper structures, prevents injury to structures lateral to the trachea e.g. the recurrent laryngeal nerves. Cosmetically disfiguring scar is not an issue with an adequate but small vertical incision. This incision renders the procedure simple for residents in training. Malata et al in their audit of Bjork flap tracheostomies in 


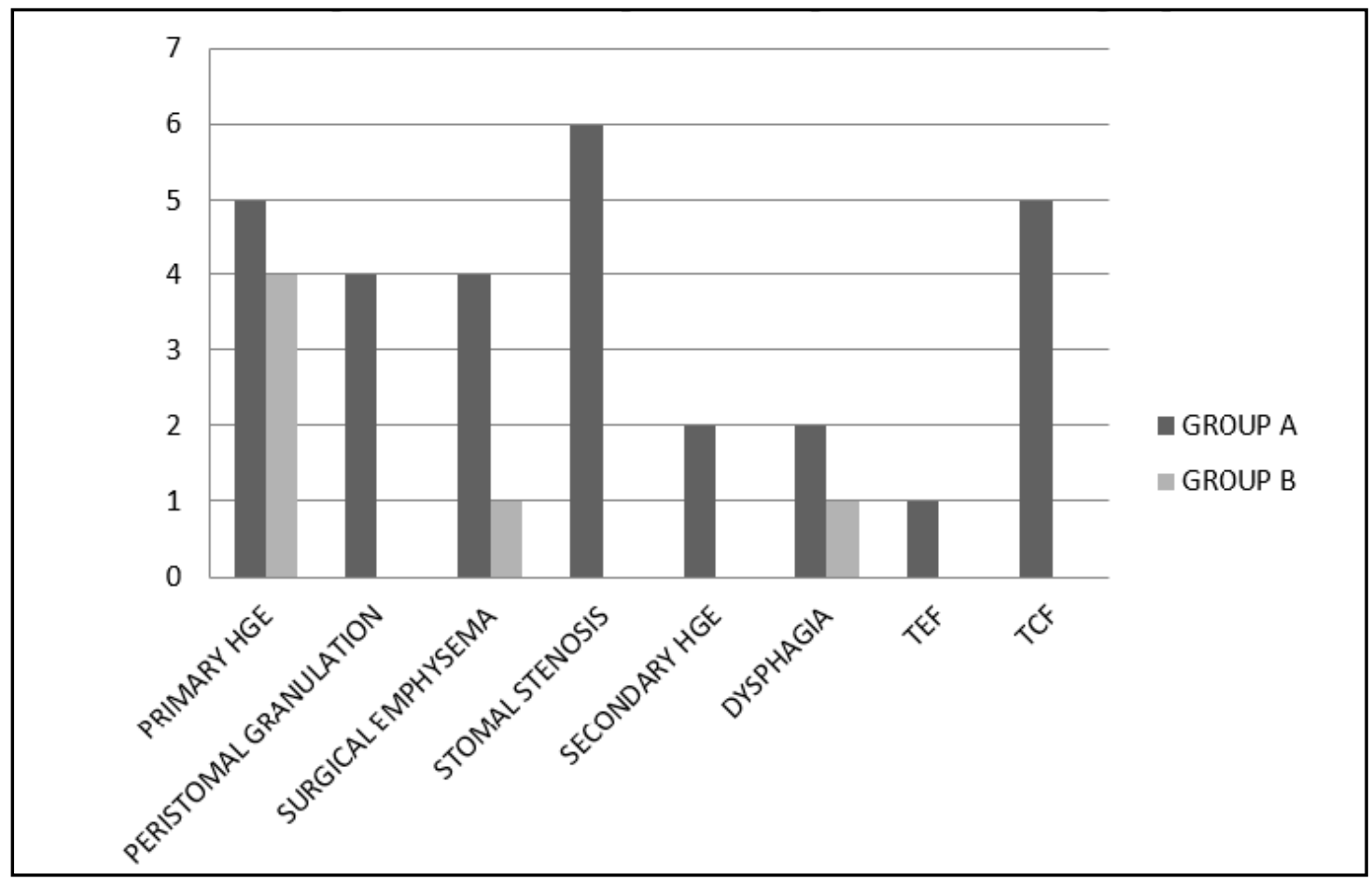

Fig. 2. Per Operative and Post Operative complications faced in either group

head and neck plastic surgery had similar opinion with regards to vertical skin incision access. ${ }^{6}$

There lies no statistical difference in the time required to access the airway in either of the groups ( $\mathrm{p}$ value $=$ 0.087). So it can be opined that Bjork flap tracheostomy can be safely undertaken in emergency situations. Weissler et al advocated Bjork flap based tracheostomy via a vertical skin incision in emergency situation. ${ }^{7}$

Flap tracheostomy advocates a flap of anterior tracheal in place of excising anterior tracheal wall window. The flap is anchored to the inferior margin of the soft tissue incision by a non absorbable suture. This ensures that the flap does not mobilise and fall back in place occluding the stoma. The tract thus created has early maturity. Its inferior surface contributed by the endotracheal mucosal lining over the flap ensures proper curved smooth passage. This enables easy tube placement. As has been the protocol in our institute the first tube change at 48 hours post operative period is done by a trained nursing personnel along with an ENT surgeon. In our study the nursing personnel could change the tube with ease without requiring any assistance from the surgeon in all the adult patients belonging to the Flap tracheostmy group i.e. Group B. But in $9.26 \%$ of the adult population in Group A has been reported to have poorly matured stoma at 48 hours and so tube changing was difficult.

Active intervention of the ENT surgeon was required to ensure tracheostomy tube placement. The trained staff had opinion that the smooth inferior wall of the matured stoma ensured that there was no false passage of the tube. The tube glided into the trachea following the endotracheal mucosal path. Considering paediatric tracheotomy (only a small incision over the anterior tracheal wall in place of excision of anterior tracheal window) there is every possibility of collapse of the tracheotomy, poor stomal maturation; flap based tracheostomy ensures preservation of tracheal wall along with prevention of the aforesaid complications. None of the paediatric patients in Group B required intervention by the surgeon during the first tube change. In Group A the single tracheostomised child required an intervention by the surgeon as the trained staff failed to cannulate the stoma on her own. 
Patients discharged with tracheostomy tube, has not only them but also their family members undergo a basic training programme for home based tracheostomy tube care and acute emergency management, before they are discharged. The family members are encouraged to care for the tracheostoma even when the patient is in hospital. This ensures supervised intervention and does away with any fear associated with home based management of tracheostomised patients. At a follow up period of one month the family members or the patients caring for the stoma were put through an assessment of the ease of care based on a Visual Analogue Scale. The ' 0 ' end denoted 'Very easy' to " 10 "- Very difficult. In Group A $16.36 \%$ of the patients reported stomal care to be "very easy". $76.36 \%$ of the patients in Group B reported it to be "very easy". Statistical correlation showed Chi square value of 39.81. $p$ value of $<0.00001$. $(<0.05=$ significant difference $)$.

So the stomal care is significantly easy in Group B as compared to Group A. Problems faced by patients or their family members in Group A include- small stoma with difficulty to put in the canula, reddish tissue growth around the opening, occasional bleeding from the tissue when the tube was forced against it, passage of the tube in place other than the airway that required replacement of the tube, need to call the local health care assistant to help place the tube. In Group B patients and family members reported easy tube placement even if accidental decannulation occurred. Heffner et al. reports that the Bjork flap provides a bridge of tracheal conduit that guides the tracheostomy tube and avoids creation of false passage and is therefore safer to use. ${ }^{8}$

Stomal healing with proper tract formation within first week is $80 \%$ in Group B as compared to $61.82 \%$ in Group A. Flap based procedure with better mucosalisation of the tract ensures early maturity of the stoma.

Complications are an important part of surgical intervention. Surgeons are faced with a larger burden of complications in emergency operative procedures like urgent airway management. In this study population, per operative haemorrhage has been an important complication. 5 and 4 patients in Group A and B respectively had primary haemorrhage. This can be attributed to manner of surgical dissection, anatomical alterations in neck, coagulopathies the patient might suffer from, the disease condition itself, or prior radiation therapy rendering the field fibrotic and difficult to work on.

Tracheo stomal closure was tried in all the paediatric patients belonging to both the groups. In adult and geriatric population it was tried in $40.74 \%$ patients in Group A and $55.77 \%$ patients in Group B. Average time of stomal closure was 2 weeks following tracheostomy. In Group A, the only child undergoing tracheotomy had resulted Tracheo-Cutaneous Fistula (TCF) following stomal closure. No child developed TCF in Group B. Due to small sample size of paediatric population, statistically significant difference amongst paediatric patients in the Groups can't be commented upon. In a study by Colman et al of 172 paediatric patients who underwent tracheostomy during a period of 4 years, no relationship between stomal maturation (as in flap tracheostomy in our study) and tracheostomy related complication- TCF. ${ }^{9} 18.18 \%$ of the adult and geriatric patients of Group A undergoing stomal closure had TCF. None of the patients in Group B had TCF following stomal closure. So there is obvious significant difference between the 2 groups. This can be attributed to the proper repositioning of the vascularised anterior tracheal wall flap back in position following decannulation ensuring prevention of TCF. According to Joseph et al age at tracheostomy and duration of tracheostomy are important determing factors for formation of Tacheocutaneous fistula. ${ }^{10}$

Tracheal steonsis at the soma, tube cuff and at the site of tubal tip is a dreaded complication of tracheostomy. Arola et al demonstrated $0-21 \%$ incidence of tracheal stenosis. ${ }^{11}$ All patients in the study population underwent evaluation of Tracheal stenosis including those who were decannulated. The evaluation was done with flexible endoscopes. In either group no cases of tracheal stenosis was found. No statistical correlation is thus impossible between the groups. Proper surgical method compounded with adequate flap width and length in Group B seems to have prevented the complication. Tracheostomy tube of adequate length, curvature and high volume low pressure cuff has also prevented the injury to tracheal mucosa along the length of the tube at the pressure points. Flap tracheostomy was studied in 25 adult canines by Lulenski et al. They used inverted $U$ 
flap. Histological examinations were made, and tracheal dimensions were calculated on sections through the stomal area. Tracheal dimensions were found to be maintained. Additional support for flap tracheostomy has been found from this animal experiment. ${ }^{12}$ Stomal stenosis had been a notorious complication in Group A although none has been reported in Flap Tracheostomy. The reason behind the finding is easy and early maturation of the tract in Group B. Inferior lip ensures easy tube placement and less chances of stoma injury in Group B as compared to A. Preistomal granulation chances are less in Group B. Thus granulations less often occlude the flap based matured stoma. Healing of immature stoma by fibrosis, traumatisation of peristomal tissue, repeated injury to the granulation during tube placement further accentuates the risk of stomal stenosis.

Complications that occurred equivocally in both the groups include- surgical emphysema and dysphagia. Complications that occurred only in Group A include peristomal granulation, secondary haemorrhage, trachea-esophageal fistula. There were no tracheostomy related deaths in either group.

\section{Conclusion}

Midline vertical soft tissue approach to any format of tracheostomy renders the process simple and is not very cosmetically disfiguring in the long run. Establishment of the airway in Bjork flap tracheostomy is equally quick making the process suited for emergency situations. Flap based tracheostomy has early stomal maturation so, care of the stoma by health assistants and family members is easy. Although there are various attributes to the formation of tracheo-cutaneous fistula, repositioning of the vascularised flap of inferior tracheal wall reduces such complications during decannulation in case of
Bjork tracheostomy. Stomal stenosis which is notorious complication of conventional method is found to be alleviated by flap tracheostomy. Rate of occurrence of other complications like peristomal granulation, surgical emphysema, and secondary haemorrhage is reduced in flap based method.

\section{References}

1. Frost EAM. Tracing the tracheostomy. Ann Otolaryngol. 1976; 85: 618-24

2. Jackson C. Tracheostomy. Laryngoscope 1909; 19: 285-90

3. Bjork VO, Engstorm CG. The treatment of ventilator insufficiency after pulmonary resection with tracheostomy and prolong artificial ventilation. J Thorasic Surg. 1955; 30:356-67

4. Kinley CE. A Technique of Tracheostomy. Can Med Assoc J. 1965; 92(2): 79-81

5. Dukes HM. Tracheostomy. Thorax 1970; 25(5): 573-6

6. Malata CM, Foo ITH, Simpson H, Batchelor AG. An audit of Bjork flap tracheostomies in head and neck plastic surgery. British J Oral Maxillofac Surg. 1996; 34: 42-6

7. Weissler MC, Couch ME. Tracheostomy and intubation. Byron. Bailey Head and Neck Sugery Otolaryngology 2006; 3: 677-89

8. Heffner JE, Miller KS, Sahn SA. Tracheostomy in the intensive care unit. Part 2: Complications. Chest 1986; 90(3): 430-6

9. Colman KL, Mandell DL, Simons JP. Impact of stoma maturation on paediatric tracheostomy related complications. Archives of Otolaryngology- Head Neck Surg. 2010; 136(5): 471-4

10. Joseph HT, Jani P, Preece JM, Bailey CM, Evans JN. Paediatric tracheostomy: persistant trachea-cutaneous fistula following decannulation. Inter J Ped Otorhinolaryngol. 1991; 22(3): 2316

11. Arola MK. Tracheostomy and its complications. A retrospective study of 794 tracheostomised patients. Ann Chir Gyneocol. 1981; 70: 96-106

12. Lulenski GC, Batsakis JG. Management of the flap tracheostomy: An experimental study. Arch of Otolaryngol. 1979; 105(5): 260-3. 